\title{
Existence Questions for the Problem of Chebyshev Approximation by Interpolating Rationals
}

\author{
By G. D. Taylor* and J. Williams
}

\begin{abstract}
This paper considers a problem of Chebyshev approximation by interpolating rationals. Examples are given which show that best approximations may not exist. Sufficient conditions for existence are established, some of which can easily be checked in practice. Illustrative examples are also presented.
\end{abstract}

1. Introduction. The problem of approximating real valued functions $f \in$ $C[a, b]$ by rationals has received considerable attention in the literature. The best known results for the classical problem of Chebyshev approximation are due to Chebyshev and Achieser (see [1]), who dealt with the problems of characterization and uniqueness of best approximations. The delicate problem of existence was successfully treated by J. L. Walsh [8].

The related problem of Chebyshev approximation by various forms of rational functions which also satisfy an interpolatory condition is of fairly recent origin; see for example Gilormini [3], Perrie [7] and Williams [9], who discuss various questions relating to existence, characterization and uniqueness. However, when dealing with the existence of best approximations, an oversight has been made by the authors in [3] and [9]. In both cases, they fail to take account of possible common zeros in the numerator and denominator of the best approximation, the effect of which is to violate the interpolation condition. Consequently, for the problems as defined by the authors, best approximations may fail to exist. For the case of the problem in [3], this was shown by a counterexample in Loeb [5]. The purpose of this paper is to present similar counterexamples for the problem as defined in [9] and to establish various sufficient conditions for the existence of best approximations. Some of these conditions can easily be checked in practice and may serve as a guide when constructing approximating functions in practical problems. The results are illustrated by simple examples.

Received February 13, 1973.

AMS (MOS) subject classifications (1970). Primary 41 A20, 41 A 30, 41 A 50, 65D20.

Key words and phrases. Existence of best rational interpolants, Chebyshev approximation, uniform rational approximation with interpolatory constraints.

*Supported in part by AFOSR 72-2271. 
2. Approximation by Interpolating Rationals, Existence and Counterexamples.

In [9], the practical problem of approximating oscillatory decay-type functions is considered and is regarded as a particular case of the following general problem. Let $D[0, b], 0<b<\infty$, denote the class of real continuous functions of the form $f(x)=$ $B(x) g(x), g \in C[0, b], g>0 \forall x \in[0, b]$, where the "oscillation" factor $B(x)$ satisfies $B\left(x_{\nu}\right)=0$ for distinct $x_{\nu} \in[0, b], \nu=1,2, \cdots, R$ (possibly a void set). Given $f \in D[0, b]$, let $V(n, p)$ denote the class of interpolating rationals of the form,

$$
F(A, x)=\frac{B(x)}{(L(A, x))^{p}}, \quad x \in[0, b]
$$

which satisfy:

(a) $L(A, x)=\sum_{r=1}^{n} a_{r} \phi_{r}(x), \phi_{1} \equiv 1, \phi_{r} \in C[0, b], r=2,3, \cdots, n$, where $\left\{\phi_{1}\right.$, $\left.\phi_{2}, \cdots, \phi_{n}\right\}$ forms a Chebyshev set on $[0, b]$;

(b) the parameter space $P$ consists of the points $A=\left(a_{1}, a_{2}, \cdots, a_{n}\right) \in E_{n}$ for which $L(A, x)>0, \quad \forall x \in[0, b]$;

(c) $0<p<\infty$.

Let $\|\cdot\|$ denote the Chebyshev norm on $[0, b]$, then $F\left(A^{*}, x\right) \in V(n, p)$ is a best Chebyshev approximation to $f$ if $\left\|f-F\left(A^{*}, x\right)\right\| \leqslant\|f-F(A, x)\| \forall F(A, x) \in$ $V(n, p)$. The crucial question here is the existence of $F\left(A^{*}, x\right) \in V(n, p)$. Throughout this paper, we shall assume that $f \notin V(n, p)$.

Two examples will now be given which show that best approximations may fail to exist. In both cases we consider the class of approximating functions $V(n, 1)$ for which $\phi_{r}(x) \equiv x^{r-1}, r=1,2, \cdots, n$. We let $R_{m}^{l}[0, b]$ denote the class of bounded rationals on $[0, b]$ of the form $\Sigma_{0}^{l} a_{r} x^{r / \Sigma_{0}} b_{r} x^{r}$.

Example 1. Let $B(x)=x(x+1), x \in[0,1]$, and select $f$ so that:

$$
\begin{aligned}
& f-9 x, \quad x \in[0,1 / 4] ; \quad f=-7 x+4, \quad x \in[1 / 4,1 / 2] \\
& f=9 x-4, \quad x \in[1 / 2,3 / 4] ; \quad f=-7 x+8, \quad x \in[3 / 4,1] .
\end{aligned}
$$

This is a "saw-tooth" function for which $|f(x)-(x+1)|=\|f-(x+1)\|=1$, at $x=$ $0,1 / 4,1 / 2,3 / 4$ and 1. Also, $f(x)-(x+1)$ alternates in sign at these points. Thus, $x+1$ is the unique best approximation to $f$ from $R_{1}^{2}[0,1]$ with error 1 . We claim that there does not exist a closest element to $f$ in $V(2,1)$. Indeed, since $V(2,1) \subset$ $R_{1}^{2}[0,1]$, we must have $\operatorname{dist}(f, V(2,1)) \geqslant 1$. Noting that $F\left(A_{n}, x\right)=$ $x(x+1) /(x+1 / n) \in V(2,1), n=1,2, \cdots$ and $\lim _{n \rightarrow \infty}\left\|f-F\left(A_{n}, x\right)\right\|=1$ (easily checked), we see that $\operatorname{dist}(f, V(2,1))=1$. This establishes our claim since $x+1$ is the only element of $R_{1}^{2}[0,1]$ satisfying $\|f-(x+1)\|=1$ and $x+1 \notin V(2,1)$.

Example 2. Let $B(x)=(x-1)^{2}, x \in[0,2]$, and set $h(x)=1 /(3-x)$. Then, similar to the above example, $f$ is the "saw-tooth" function obtained by linking with straight lines the points, in order, whose coordinates are: 


$$
\begin{aligned}
& \left(0, h(0)+\frac{1}{2}\right),\left(1, h(1)-\frac{1}{2}\right),\left(\frac{6}{5}, h\left(\frac{6}{5}\right)+\frac{1}{2}\right),\left(\frac{7}{5}, h\left(\frac{7}{5}\right)-\frac{1}{2}\right), \\
& \left(\frac{8}{5}, h\left(\frac{8}{5}\right)+\frac{1}{2}\right),\left(\frac{9}{5}, h\left(\frac{9}{5}\right)-\frac{1}{2}\right),\left(2, h(2)+\frac{1}{2}\right) .
\end{aligned}
$$

$f(x)$ satisfies $|f(x)-h(x)|=\|f-h\|=1 / 2$, at $x=0,1,6 / 5,7 / 5,8 / 5,9 / 5$ and 2. Also, $f(x)-$ $h(x)$ alternates in sign at these points. Consider approximation of $f$ by the class $V(4,1)$ and set

$$
F\left(A_{n}, x\right)=\frac{(x-1)^{2}}{\left((x-1)^{2}+1 / n^{2}\right)(3-x)} \in V(4,1), \text { for } n=1,2, \cdots .
$$

As above, $1 /(3-x)$ is the best approximation to $f$ from $R_{3}^{2}[0,2]$ with error $1 / 2$ and $F\left(A_{n}, x\right) \in R_{3}^{2}[0,2], n=1,2, \cdots$, satisfies $\lim _{n \rightarrow \infty}\left\|f-F\left(A_{n}, x\right)\right\|=1 / 2$. Thus, no best approximation to $f$ from $V(4,1)$ exists.

It is clear that similar examples of nonexistence can be constructed for the class $V(n, p)$ with $p>1$.

In view of the possible failure of existence, it is natural to seek conditions on $f(x)=B(x) g(x)$ which guarantee the existence of a best approximation. This will be approached in two ways. First, conditions on $B(x)$ will be described and then, conditions in terms of $g(x)$ will be established.

3. Existence of Best Approximations, Conditions on $B(x)$. In the following theorem, we give a sufficient condition for best approximations from $V(n, p)$ to exist; that is, a restriction is placed on the "orders" of the zeros of $B(x)$ relative to the size of the positive parameter $p$. Roughly speaking, the sufficient condition for existence is that interior zeros of $B$ have "order" less than $2 p$; and zeros of $B$ at the endpoints have "order" less than $p$ (here $p$ need not be an integer). In particular, if $B$ has no zeros, then existence is assured.

THEOREM 3.1. Let $B(x)$ have zeros $x_{\nu} \in[0, b], \nu=1,2, \cdots, R$ (possibly a void set) which satisfy,

(i) for each $r=2, \cdots, n, \phi_{r}$ is analytic in open discs with centres at the interior zeros $x_{\nu} \in(0, b)$;

(ii) $\lim _{x \rightarrow x_{\nu^{ \pm}}}\left|B(x) /\left(x-x_{\nu}\right)^{p \mid}\right|=+\infty$ if $x_{\nu}=0$ or $x_{\nu}=b$;

(iii) $\lim _{x \rightarrow x_{\nu}}\left|B(x) /\left(x-x_{\nu}\right)^{2 p}\right|=+\infty$ if $x_{\nu} \in(0, b)$.

Then, for all $f(x)=B(x) g(x) ; g \in D[0, b]$, there exist best approximations in $V(n, p)$.

Proof. Using an elementary argument presented in [9], it can be shown that for $f \in D[0, b]$ a best approximation, if one exists, must lie in the parameter set

$$
P_{M}:=\{A:\|f-F(A, x)\| \leqslant\|f\|-\eta\} \subset P,
$$

for some $\eta>0$. Also, it can be shown that the sequence $\left\{A_{k}\right\}$ in $P_{M}$ given by

$$
\lim _{k \rightarrow \infty}\left\|f-F\left(A_{k}, x\right)\right\|=\inf _{A \in \mu_{M}}\|f-F(A, x)\|
$$


satisfies $\left\|L\left(A_{k}, x\right)\right\| \leqslant K \quad \forall k$ and for some $K<\infty$. Because of compactness, there exists a subsequence $\left\{L\left(A_{j}, x\right)\right\}$ of $\left\{L\left(A_{k}, x\right)\right\}$ for which $L\left(A_{j}, x\right) \rightarrow L\left(A^{*}, x\right)$ uniformly. In order to show that $F\left(A^{*}, x\right) \in V(n, p)$, it is necessary to examine the zeros of $L\left(A^{*}, x\right)$. Since $\left\|F\left(A_{j}, x\right)\right\| \leqslant K_{1} \forall j$ and some $K_{1}<\infty$, it follows that, if there exists $t \in[0, b]$ for which $L\left(A^{*}, t\right)=0$, then $t=x_{\nu}$ for some $\nu$. Condition (ii) further implies that $t \neq 0$ (or $t \neq b$ ).

Suppose, noting condition (i), that, for some $t=x_{\nu} \in(0, b), L\left(A^{*}, x_{\nu}\right)=0$ with multiplicity $m_{\nu}$. Rouche's theorem implies that for a given $\epsilon>0$, sufficiently small (and depending on condition (i)), there exists $N(\epsilon)$ such that, for $j \geqslant N, L\left(A_{j}, z\right)$ has exactly $m_{\nu}$ zeros in the disc $\left|z-x_{\nu}\right| \leqslant \epsilon$. Now, if for some complex-valued $\alpha$ in this $\operatorname{disc} L\left(A_{j}, \alpha\right)=0$, then $L\left(A_{j}, \bar{\alpha}\right)=0$ by the Schwartz reflection principle. Hence, $L\left(A^{*}, z\right)$ has a zero of multiplicity at least two at $x_{\nu}$. But this is impossible because of condition (iii). Thus, $L\left(A_{j}, z\right)$ must have a real zero in the disc so that $A_{j} \notin P_{M}$. Therefore, $L\left(A^{*}, x\right)$ cannot have a zero at $x_{\nu} \in(0, b)$ so that $F\left(A^{*}, x\right) \in$ $V(n, p) ; F\left(A^{*}, x\right)$ is thus a best approximation and the proof is complete.

Examples. (See [9].)

In each case, we consider the class $V(n, p)$ for which $\phi_{r}(x)=x^{r-1}, r=1$, $2, \cdots, n$, and give, in the form of lower bounds, conditions on $p$ which are sufficient for existence.

1. $f(x)=10 x^{2}(1-x) e^{-2 x}, B(x)=x^{2}(1-x), x \in[0,1]$. Best approximations to $f$ exist in $V(n, p)$ for $p>2$. Similarly for the problem of best approximation on $[0,20]$.

2. $f(x)=-1 / \Gamma(x-3), B(x)=x(1-x)(2-x)(3-x), x \in[0,10]$. Best approximations to $f$ exist in $V(n, p)$ for $p>1$.

4. Existence of Best Approximations, Conditions on $g(x)$. In [9], characterization (in the form of a modified alternation property) and uniqueness of a best approximation are established using the results of Meinardus and Schwedt [6]. With the aid of these results, an exchange algorithm is also described for computing best approximations. This consists of computing the sequence $F\left(A_{k}, x\right) \in V(n, p), k=0,1$, $2, \cdots$, where $F\left(A_{k}, x\right)$ is a best approximation to $f$ on the reference $X(k):=\left\{x_{1}^{(k)}\right.$, $\left.\cdots, x_{n+1}^{(k)}\right\} \subset[0, b] . F\left(A_{k}, x\right)$ is obtained by solving the nonlinear equations

$$
\left|B\left(x_{r}^{(k)}\right)\right|\left\{g\left(x_{r}^{(k)}\right)-L\left(A_{k}, x_{r}^{(k)}\right)^{-p}\right\}=(-)^{r} \lambda_{k}, \quad r=1,2, \cdots, n+1,
$$

where $\left|\lambda_{k}\right|$ is the error of the approximation on $X^{(k)}$.

The next stage of the algorithm consists of selecting a new reference $X^{(k+1)}$ from the extrema of the error function $f(x)-F\left(A_{k}, x\right)$. This is always possible. Since $f \notin V(n, p)$, a zero $x_{\nu}$ of $B(x)$ cannot coincide with a reference point. The uniform convergence of $\left\{F\left(A_{k}, x\right)\right\}$ to the best approximation $F\left(A^{*}, x\right)$ can now be estab- 
lished [9]. In the proof, it is not necessary to assume the existence of a best approximation; all that is required is that for each $k$ there exists a solution of (4.1) which satisfies $F\left(A_{k}, x\right) \in V(n, p)$. In considering Eqs. (4.1), properties of Chebyshev sets are used in [9] to prove

THEOREM 4.1. For an arbitrary reference $X:=\left\{x_{r}\right\} \subset[0, b], B\left(x_{r}\right) \neq 0, r=$ $1,2, \cdots, n+1$, there exists a unique real solution $(A, \lambda)$ of Eqs. (4.1). The resulting approximation satisfies $L\left(A, x_{r}\right)>0$ for $r=1,2, \cdots, n+1$.

In practice, it is necessary to assume that the solution of (4.1) satisfies $F\left(A_{k}, x\right) \in$ $V(n, p) \forall k$. If, however, conditions on $g(x)$ can be introduced which ensure that $F\left(A_{k}, x\right) \in V(n, p) \forall k$, then existence of best approximations is guaranteed for the class of functions $f(x)=B(x) g(x) \in D[0, b]$.

THEOREM 4.2. Let $L(A, x)$ interpolate $h(x)=g(x)^{-1 / p}$ at the distinct points $X:=0 \leqslant x_{1}<x_{2}<\cdots<x_{n} \leqslant b$, so that $A$ is uniquely determined by

$$
L\left(A, x_{r}\right)=h\left(x_{r}\right), \quad r=1,2, \cdots, n .
$$

If for all such sets of interpolating points $X \subset[0, b]$, there exist $\eta>0$ such that

$$
L(A, x) \geqslant \eta \quad \forall x \in[0, b],
$$

then, for all $f(x)=B(x) g(x) \in D[0, b]$ there exist best approximations in $V(n, p)$.

Proof. We shall show that for such functions $f(x)=B(x) g(x)$ each step of the exchange algorithm yields a unique approximation $F\left(A_{k}, x\right) \in V(n, p)$; existence of $F\left(A^{*}, x\right) \in V(n, p)$ thus follows.

For an arbitrary reference $X:=\left\{x_{r}\right\}, B\left(x_{r}\right) \neq 0$, Theorem 4.1 shows that Eqs. (4.1) have a unique solution $(A, \lambda)$ which satisfies:

$$
L\left(A, x_{r}\right)^{p}-\frac{1}{g\left(x_{r}\right)}=\frac{(-1)^{r} \lambda L\left(A, x_{r}\right)^{p}}{\left|f\left(x_{r}\right)\right|}, \quad r=1,2, \cdots, n+1 .
$$

Hence, by continuity, there exist $n$ points $x_{1}<z_{1}<z_{2}<\cdots<z_{n}<x_{n+1}$ for which

$$
L\left(A, z_{r}\right)=h\left(z_{r}\right), \quad r=1,2, \cdots, n,
$$

so that, by hypothesis, $L(A, x) \geqslant \eta \forall x \in[0, b]$; thus $F(A, x) \in V(n, p)$.

The following two corollaries and examples apply to the class $V(n, p)$ where $\phi_{r} \equiv x^{r-1}, r=1,2, \cdots, n$.

Corollary 1. Let $h \in C^{\infty}[0, b]$ with $\left\|h^{(r)}\right\| \leqslant M^{r} \forall r>1$ and some $M \geqslant$ 0 . Then there exists $N$ such that, for all $f(x)=B(x) g(x) \in D[0, b]$, best approximations exist in $V(n, p)$ for $n>N$.

Proof. Let $L(A, x)$ interpolate $h(x)$ at the arbitrary distinct points $x_{i} \in$ $[0, b], i=1,2, \cdots, n$; then, from the error in Lagrangian interpolation,

$$
\|h-L(A, x)\| \leqslant(M b)^{n} / n ! .
$$

Since $h>0 \quad \forall x \in[0, b]$, the result follows from Theorem 4.2.

COROLlARY 2. Let $h$ be analytic on the closed region of the complex plane 
consisting of all points within distance $b$ of the interval $[0, b]$. Then there exists $N$ such that, for $f=B(x) g(x) \in D[0, b]$ best approximations exist in $V(n, p)$ for . $n>N$.

Proof. The proof follows as in Corollary 1 where now we use the contour integral form of the interpolation error [4, p. 23].

Examples. The following examples follow easily from the proof of Corollary 1 .

1. $f(x)=10 x^{2}(1-x) e^{-2 x}, p=1, g(x)=10 e^{-2 x}, x \in[0,1]$. Here $h(x)=e^{2 x / 10}$ and, for positive interpolating polynomials $L(A, x)$, it is sufficient to have

$$
\|h-L(A, x)\|<\frac{1}{10}
$$

But from the error in Lagrangian interpolation,

$$
\|h-L(A, x)\| \leqslant \frac{1}{n !} \frac{1}{10} 2^{n} e^{2}
$$

and so (1) will be satisfied for all $n>5$. Thus, best approximations to $f$ exist in $V(n, 1)$ for $n>5$. This result provides an extension to Example 1 of Section 3 .

2. $f(x)=x(x-1 / 2)^{3}(x-1)^{2} / \sin (x+\pi / 6), p=1, g(x)=1 / \sin (x+\pi / 6), x \in[0,1]$.

Best approximations to $f$ exist in $V(n, 1)$ for $n>1$. We note here that $B(x)$ does not satisfy conditions (ii) and (iii) of Theorem 3.1.

5. Concluding Remarks. Finally, it is of some practical interest to consider the above approximation problems on $[0, \infty)$. Here we wish to add a word of caution about expecting to be able to use the exchange algorithm to compute best approximations. This technique can fail since the characterization theorem (see [9]) may fail to hold in this setting. That this is so follows from the work of Brink [2] who considered the special case of approximating $f(x)=g(x) \in D[0, \infty), f \in C^{\infty}[0, \infty), \lim _{x \rightarrow \infty} f(x)=0$, by elements in $V(n, 1), \phi_{r} \equiv x^{r-1}, r=1,2, \cdots, n$. In this study, it is shown that best approximations are completely characterized by one of two possible alterna tion conditions holding (one of which is the standard alternation condition).

\section{Mathematics Department \\ Colorado State University \\ Fort Collins, Colorado 80521 \\ Department of Mathematics \\ University of Manchester \\ Manchester, M13 9PL, England}

1. N. I. AHIEZER, Lectures on the Theory of Approximation, Kharkov, 1940; English transl., Ungar, Ne'v York, 1956. MR 3, 234; 20 \#1872.

2. D. BRINK, Tchebycheff Approximation by Reciprocals of Polynomials on $[0, \infty]$, Thesis, Michigan State University, East Lansing, Mich., 1972.

3. C. GILORMINI, "Approximation rationelle avec des noeuds," C. R. Acad. Sci. Paris Sér. $A-B$, v. 263, 1966, pp. A286-A287. MR $34 \# 4768$. 1966.

4. D. C. HANDSCOMB, Methods of Numerical Approximation, Pergamon Press, London,

5. H. L. LOEB, "Un contre-exemple à un résultat de M. Claude Gilormini," C. R. Acad. Sci. Paris Sér. $A-B$, v. 266, 1968, pp. A237-A238. MR 37 \#578. 
6. G. MEINARDUS, Approximation of Functions: Theory and Numerical Methods, Springer Tracts in Natural Philosophy, vol. 13, Springer-Verlag, New York, 1967. MR 36 \#571.

7. A. L. PERRIE, "Uniform rational approximation with osculatory interpolation," J. Comput. System Sci, v. 4, 1970 , pp. 509-522. MR 43 \#776.

8. J. L. WALSH, "The existence of rational functions of best approximation," Trans. Amer. Math. Soc., v. 33, 1931 , pp. 668-689.

9. J. WILLIAMS, "Numerical Chebyshev approximation by interpolating rationals," Math. Comp., v. 26, 1972, pp. 199-206. 